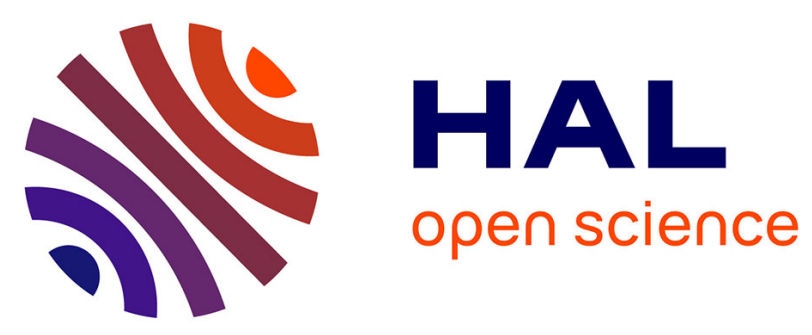

\title{
Conjecture on the critical frontier of the Potts ferromagnet on planar lattices
}

C. Tsallis, A.C.N. de Magalhães

\section{To cite this version:}

C. Tsallis, A.C.N. de Magalhães. Conjecture on the critical frontier of the Potts ferromagnet on planar lattices. Journal de Physique Lettres, 1981, 42 (11), pp.227-231. 10.1051/jphyslet:019810042011022700 . jpa-00231915

\section{HAL Id: jpa-00231915 https://hal.science/jpa-00231915}

Submitted on 1 Jan 1981

HAL is a multi-disciplinary open access archive for the deposit and dissemination of scientific research documents, whether they are published or not. The documents may come from teaching and research institutions in France or abroad, or from public or private research centers.
L'archive ouverte pluridisciplinaire HAL, est destinée au dépôt et à la diffusion de documents scientifiques de niveau recherche, publiés ou non, émanant des établissements d'enseignement et de recherche français ou étrangers, des laboratoires publics ou privés. 


\title{
Conjecture on the critical frontier of the Potts ferromagnet on planar lattices
}

\author{
C. Tsallis and A. C. N. de Magalhães \\ Centro Brasileiro de Pesquisas Físicas/CNPq, Av. Wenceslau Braz, 71, Rio de Janeiro, Brazil
}

(Reçu le 6 février 1981, révisé le 26 mars, accepté le 7 avril 1981)

\begin{abstract}
Résumé. - On propose une conjecture sur les frontières critiques approchées du modèle ferromagnétique de Potts à $q$ états sur des réseaux plans. Cette conjecture est vérifiée avec une précision satisfaisante pour une variété de réseaux plans (et aussi pour le réseau carré avec premiers et seconds voisins), et permet de prédire un nombre considérable de résultats nouveaux ( 29 points critiques indépendants et quelques lignes critiques).
\end{abstract}

\begin{abstract}
A conjecture is proposed for the approximate critical frontiers of the $q$-state Potts ferromagnets on planar lattices. This conjecture is verified, within a satisfactory degree of accuracy, for a variety of planar lattices (as well as for the first and second neighbour square one), and enables the prediction of a considerable number of new results (29 independent critical points and a few critical lines).
\end{abstract}

It is well known that the ferromagnetic $q$-state Potts model presents a second-order (first-order) phase transition for all dimensionalities $d>1$ and number of states $q \leqslant q_{\mathrm{c}}(d)\left(q>q_{\mathrm{c}}(d)\right)$; in particular $q_{\mathrm{c}}(2)=4$. Note that $q=2$ corresponds to the $1 / 2$-spin Ising model, and the $q \rightarrow 1$ and $q \rightarrow 0$ limits correspond respectively to the standard and tree-like bond percolations $[1,2]$. The critical frontiers associated with the anisotropic square, triangular and honeycomb lattices are at present, as far as we know, the only ones which are exactly known [3] for all $q$, while, of course, several other planar lattices have been solved for $q=2$ [4]. In the present work we make a conjecture which essentially states that knowledge, for a particular value of $q(1 \leqslant q \leqslant 4)$, of the simple [5] paramagnetic-ferromagnetic critical frontier (CF) associated with any given planar lattice (isotropic or not, homogeneous or not) enables one to calculate, at least approximately, the critical frontier associated with the same lattice for the other values of $q(1 \leqslant q \leqslant 4)$.

Let us consider a single Potts bond (coupling constant $J)$; it is convenient to introduce a variable $t^{(q)}$ (referred hereafter as thermal transmissivity, see references [6-8] and references therein) defined as follows

$$
t^{(q)} \equiv \frac{1-\mathrm{e}^{-q J / k_{B} T}}{1+(q-1) \mathrm{e}^{-q J / k_{B} T}} \quad(J>0) .
$$

It is interesting to remark that the $t^{(q)}$-variable precisely coincides, for all $q$, with the $p$-variable Stephen [2] found useful to work with. Let us stress that $t^{(1)}=1-\mathrm{e}^{-J / k_{\mathrm{B}} T}$ is precisely the variable isomorphic [1] to the occupation probability of the standard bond percolation problem. It is straightforward to verify [8] that the equivalent transmissivity $t_{\mathbf{s}}^{(q)}$ of a series array of two bonds with transmissivities $t_{1}^{(q)}$ and $t_{2}^{(q)}$ is given by

$$
t_{\mathrm{s}}^{(q)}=t_{1}^{(q)} t_{2}^{(q)}
$$

whereas for a parallel array it is

$$
t_{\mathbf{p}}^{(q) \mathbf{D}}=t_{1}^{(q) \mathbf{D}} t_{2}^{(q) \mathbf{D}}
$$

where we have introduced [8] the dual [9] transmissivity

$$
t_{i}^{(q) \mathrm{D}} \equiv \frac{1-t^{(q)}}{1+(q-1) t^{(q)}} \quad \forall i
$$

Let us now introduce [7] a new variable $s^{(q)}$ through the relation

$$
s^{(q)} \equiv \frac{\ln \left[1+(q-1) t^{(q)}\right]}{\ln q} .
$$

We verify a remarkable property, namely,

$$
s^{(q)}\left\{t^{(q) \mathrm{D}}\right\}=1-s^{(q)}\left\{t^{(q)}\right\}
$$


i.e. the $s$-variable transforms, under duality, like a probability. We verify as well that, in the limit $q \rightarrow 1$, $s^{(1)}=t^{(1)}$. We are now prepared to state our conjecture. We shall assume known, for a certain planar lattice, the CF for a fixed value $q_{0}\left(1 \leqslant q_{0} \leqslant q_{\mathrm{c}}(2)\right)$, namely, the equation

$$
\varphi\left\{s_{1}^{\left(q_{0}\right)}, s_{2}^{\left(q_{0}\right)}, \ldots, s_{n}^{\left(q_{0}\right)}\right\}=0
$$

where $s_{1}^{\left(q_{0}\right)}, s_{2}^{\left(q_{0}\right)}, \ldots, s_{n}^{\left(q_{0}\right)}$ are associated with the independent coupling constants $J_{1}, J_{2}, \ldots, J_{n}$ of the system under consideration. Our conjectural statement will be that the equation

$$
\varphi\left\{s_{1}^{(q)}, s_{2}^{(q)}, \ldots, s_{n_{-}}^{(q)}\right\}=0
$$

represents also, either exactly or within great accuracy, the CF of the corresponding $q$-state Potts model for the other values of $q\left(1 \leqslant q \leqslant q_{\mathrm{c}}(2)\right)$.

1. Anisotropic square lattice. - The bond percolation CF for this lattice is given by [10]

$$
p_{1}+p_{2}=1
$$

which, within the present framework, will be generalized into

$$
s_{1}^{(q)}+s_{2}^{(q)}=1
$$

which reproduces the exact [3] critical temperature for any value of $q$. We see, therefore, that for this system the present conjecture is rigorously true $(0 \leqslant q \leqslant 4)$. Moreover, this might happen only for this lattice, as a consequence of its self-duality.

2. Anisotropic triangular and honeycomb lattices. The bond percolation CF frontier for the anisotropic triangular lattice is given by [10]

$$
1-p_{1}-p_{2}-p_{3}+p_{1} p_{2} p_{3}=0
$$

(for the honeycomb lattice -ditto with $p_{i} \rightarrow 1-p_{i} \forall i$ ). Therefore, within the present framework, the CF for any value of $q(1 \leqslant q \leqslant 4)$ is approached by

$$
1-s_{1}^{(q)}-s_{2}^{(q)}-s_{3}^{(q)}+s_{1}^{(q)} s_{2}^{(q)} s_{3}^{(q)}=0 .
$$

Let us now compare this equation with the exact [3] one. We immediately verify that they coincide whenever one of the three coupling constants vanishes (anisotropic square lattice limit). Next we perform the comparison for the maximal error case, namely, the isotropic limit. Our conjecture leads to

$$
s^{(q)}=2 \sin \frac{\pi}{18}
$$

for this lattice, whereas the exact answer is given by

$\frac{t^{(q)}}{1-t^{(q)}}=\frac{2}{q} \cos \left[\frac{1}{3} \arccos \left(\frac{q}{2}-1\right)\right]-\frac{1}{q}$

$$
(q \leqslant 4) \text {. }
$$

The results are presented in figure 1 . We note that the error is smaller than $2.4 \%$ for $1 \leqslant q \leqslant 4$. It is straightforward to verify [11] that, in the $q \rightarrow 0$ limit, $t^{(q)} \sim 1-L \sqrt{q}$, hence $s^{(q)} \sim \frac{1}{2}+\frac{\ln L}{\ln q}$, where $L$ is a lattice-dependent pure number $(L=1, \sqrt{3}$, $1 / \sqrt{3}$ for the square, triangular and honeycomb lattices respectively; as a matter of fact for any pair of dual lattices it holds $L^{\mathrm{D}}=1 / L$ ). The non-vanishing error comes from the tendency of $s^{(q)}$ to approach $1 / 2$, and its small size from the infinite slope at $q=0$.

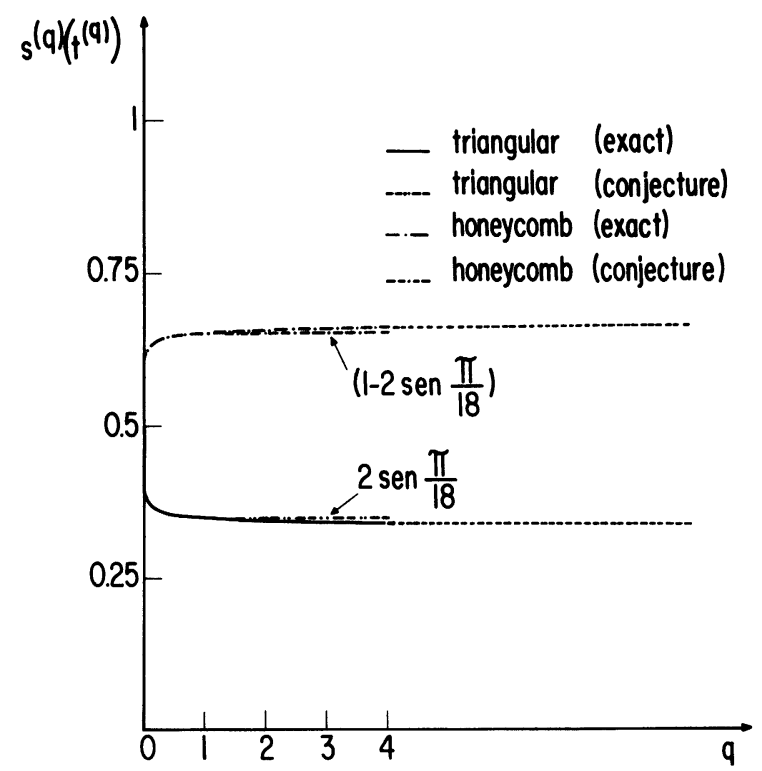

Fig. 1. - Comparison between the exact and conjectural critical points for the isotropic triangular and honeycomb lattices (the analytic extension for $q>4$ is also shown).

3. «Inhomogeneous » 4-8 lattice and its dual. The exact CF's associated with the Ising model in the « inhomogeneous» 4-8 lattice (Fig. 10 of reference [4]) and its dual (square lattice with non-crossing diagonal bonds) are known [4]. Since there is a straightforward relation between the CF's of any pair of dual lattices, we shall restrict our discussion here to the 4-8 lattice (we note that $J_{1}$ and $J_{2}$, the coupling constants associated with the different bonds are in a $2: 1$ ratio). The exact CF in the $s_{1}^{(2)}-s_{2}^{(2)}$ space is represented in figure 2 . We may verify that

$$
\begin{aligned}
& s_{1}^{(2)}=s_{2}^{(2)} \simeq 0.6792, \\
&\left.\frac{\mathrm{d} s_{1}^{(2)}}{\mathrm{d} s_{2}^{(2)}}\right|_{s_{2}^{(2)}=1} \simeq-0.414
\end{aligned}
$$

and

$$
\left(s_{2}^{(2)}-\frac{1}{2}\right) \sim A\left(s_{1}^{(2)}-1\right)^{2} \quad(A \simeq 1.39) .
$$

In the present framework, this CF should, within satisfactory accuracy, be the same for $q \neq 2$; let us 
Table I. - Critical points for isotropic and homogeneous $q$-state Potts ferromagnets in a set of lattices. $t^{(q)}$ and $s^{(q)}$ are related to $k_{\mathrm{B}} T_{\mathrm{c}} / J$ through equations $(1)$ and $(5) ;(.$.$) denotes an exact value. For the triangular and honey-$ comb lattices the exact, rather than the conjectural, values are indicated. The region delimited by a heavy line contains results that, as far as we know, have not yet been checked by any other procedure. * This central value has been adopted after consideration of the Ising critical lines associated with the case where the first and second coupling constants are not necessarily equal.

\begin{tabular}{|c|c|c|c|c|c|c|c|}
\hline \multirow{2}{*}{ Lattices } & \multirow{2}{*}{$\begin{array}{c}q=1 \\
s^{(1)}=t^{(1)}\end{array}$} & \multicolumn{2}{|c|}{$q=2$} & \multicolumn{2}{|c|}{$q=3$} & \multicolumn{2}{|c|}{$q=4$} \\
\hline & & $s^{(2)}$ & $t^{(2)}$ & $s^{(3)}$ & $t^{(3)}$ & $s^{(4)}$ & $t^{(4)}$ \\
\hline Square & $1 / 2[10]$ & $1 / 2$ & $0.414 \ldots[4]$ & $1 / 2$ & $0.366 \ldots[3]$ & $1 / 2$ & $1 / 3[3]$ \\
\hline Triangular & $0.347 \ldots[10]$ & $0.342 \ldots$ & $0.268 \ldots[4]$ & $0.340 \ldots$ & $0.227 \ldots[3]$ & $0.339 \ldots$ & $1 / 5[3]$ \\
\hline Honeycomb & $0.653 \ldots[10]$ & $0.658 \ldots$ & $0.577 \ldots[4]$ & $0.660 \ldots$ & $0.532 \ldots[3]$ & $0.661 \ldots$ & $1 / 2[3]$ \\
\hline $4-8$ & $\begin{array}{l}0.679 \pm 0.006 \\
0.680 \pm 0.005[12] \\
0.675 \pm 0.027[13] \\
0.684[14] \\
{[0.645,0.707][15]}\end{array}$ & $0.679 \ldots$ & $0.601 \ldots[4]$ & $0.679 \pm 0.003$ & $0.554 \pm 0.003$ & $0.679 \pm 0.005$ & $0.521 \pm 0.006$ \\
\hline $\begin{array}{l}\text { Non-crossing dia- } \\
\text { gonal square-lat- } \\
\text { tice }\end{array}$ & $0.321 \pm 0.006$ & $0.321 \ldots$ & $0.249 \ldots[4]$ & $0.321 \pm 0.003$ & $0.211 \pm 0.002$ & $0.321 \pm 0.005$ & $0.187 \pm 0.004$ \\
\hline Kagomé & $\begin{array}{l}0.521 \pm 0.006 \\
0.526[14] \\
{[0.522,0.529][15]}\end{array}$ & $0.521 \ldots$ & $0.435 \ldots[4]$ & $0.521 \pm 0.003$ & $0.387 \pm 0.003$ & $0.521 \pm 0.005$ & $0.354 \pm 0.005$ \\
\hline Diced & $0.479 \pm 0.006$ & $0.479 \ldots$ & $0.393 \ldots[4]$ & $0.479 \pm 0.003$ & $0.346 \pm 0.003$ & $0.479 \pm 0.005$ & $0.314 \pm 0.004$ \\
\hline $3-12$ & $\begin{array}{l}0.740 \pm 0.011 \\
0.751[14]\end{array}$ & $0.740 \ldots$ & $0.671 \ldots[4]$ & $0.740 \pm 0.005$ & $0.628 \pm 0.006$ & $0.740 \pm 0.008$ & $0.597 \pm 0.010$ \\
\hline Asanoha & $0.260 \pm 0.011$ & $0.260 \ldots$ & $0.197 \ldots[4]$ & $0.260 \pm 0.005$ & $0.165 \pm 0.004$ & $0.260 \pm 0.008$ & $0.144 \pm 0.005$ \\
\hline$(4,6,12)$ & $0.693[14]$ & $0.693 \pm 0.012$ & $0.617 \pm 0.013$ & $0.693 \pm 0.017$ & $0.571 \pm 0.020$ & $0.693 \pm 0.021$ & $0.538 \pm 0.025$ \\
\hline$(3,4,6,4)$ & $0.525[14]$ & $0.525 \pm 0.012$ & $0.439 \pm 0.012$ & $0.525 \pm 0.017$ & $0.390 \pm 0.017$ & $0.525 \pm 0.021$ & $0.357 \pm 0.020$ \\
\hline$(3,3,3,3,6)$ & $0.439[14]$ & $0.439 \pm 0.012$ & $0.356 \pm 0.011$ & $0.439 \pm 0.017$ & $0.310 \pm 0.015$ & $0.439 \pm 0.021$ & $0.279 \pm 0.018$ \\
\hline $\begin{array}{c}(3,3,3,4,4) \text { and } \\
(3,3,4,3,4)\end{array}$ & $0.422[14]$ & $0.422 \pm 0.012$ & $0.340 \pm 0.011$ & $0.422 \pm 0.017$ & $0.295 \pm 0.015$ & $0.422 \pm 0.021$ & $0.265 \pm 0.017$ \\
\hline $\begin{array}{l}\text { 1st and 2nd Neigh- } \\
\text { bour square lat- } \\
\text { tice }\end{array}$ & $\left\{\begin{array}{l}0.249 \pm 0.011 \\
0.250 \pm 0.003[12]\end{array}\right.$ & $0.249 \pm 0.007$ & $\begin{array}{l}0.188 \pm 0.006^{*} \\
0.189[16] \\
0.188[17] \\
0.184[18] \\
0.199[19]\end{array}$ & $0.249 \pm 0.008$ & $0.157 \pm 0.006$ & $0.249 \pm 0.009$ & $0.137 \pm 0.006$ \\
\hline $\begin{array}{l}\text { 1st and } 2 \text { nd Neigh- } \\
\text { bour triangular } \\
\text { lattice }\end{array}$ & $0.155 \pm 0.004$ & $0.155 \pm 0.001$ & $\begin{array}{l}0.1135 \pm 0.0010 \\
0.1135[17] \\
0.1131[23]\end{array}$ & $0.155 \pm 0.002$ & $0.093 \pm 0.001$ & $0.155 \pm 0.003$ & $0.080 \pm 0.002$ \\
\hline $\begin{array}{l}\text { 1st, 2nd and 3rd } \\
\text { Neighbour square } \\
\text { lattice }\end{array}$ & $0.154 \pm 0.004$ & $0.154 \pm 0.001$ & $\begin{array}{l}0.1130 \pm 0.0010 \\
0.1130[23]\end{array}$ & $0.154 \pm 0.002$ & $0.092 \pm 0.001$ & $0.154 \pm 0.003$ & $0.080 \pm 0.002$ \\
\hline $\begin{array}{l}\text { 1st, 2nd and 3rd } \\
\text { Neighbour trian- } \\
\text { gular lattice }\end{array}$ & $0.099 \pm 0.003$ & $0.099 \pm 0.001$ & $\begin{array}{l}0.0711 \pm 0.0010 \\
0.0711[23]\end{array}$ & $0.099 \pm 0.002$ & $0.057 \pm 0.001$ & $0.099 \pm 0.002$ & $0.049 \pm 0.001$ \\
\hline
\end{tabular}




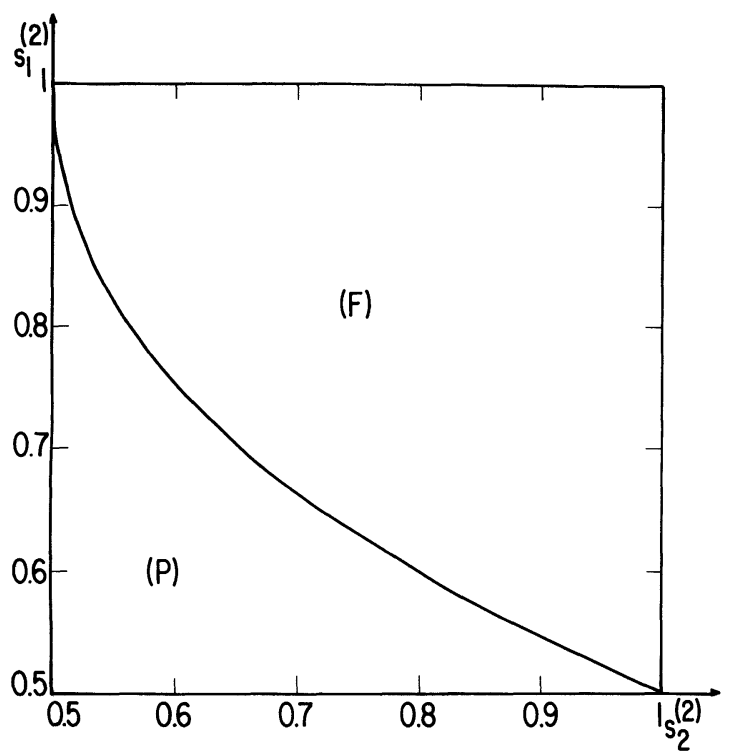

Fig. 2. - The exact para (P) - ferro (F) - magnetic critical frontier of the 4-8 lattice Ising model (as a matter of fact the proposed [12] $q=1$ critical line is indistinguishable from the $q=2$ one, within the present scale).

compare it with a recent conjecture [12] (completely unrelated to the present one) for bond percolation in the same system, namely

$$
3\left(s_{2}^{(1)}-1 / 2\right)-4\left[\left(1-s_{1}^{(1)}\right)^{2}+\left(1-s_{1}^{(1)}\right)^{3}\right]=0 .
$$

From this equation it results that $s_{1}^{(1)}=s_{2}^{(1)} \simeq 0.6801$ (which compares well with $0.675 \pm 0.027$ [13] and 0.684 [14], lies within the conjectural interval $0.645-0.707$ [15], and which differs from the value 0.6792 by only $0.13 \%$ ),

$$
\mathrm{d} s_{1}^{(1)} /\left.\mathrm{d} s_{2}^{(1)}\right|_{s_{2}^{(1)=1}}=-3 / 7 \simeq-0.429
$$

(the discrepancy with the value -0.414 is $3.5 \%$ ), and the same asymptotic behaviour mentioned previously for $q=2$ is satisfied with $A=4 / 3$ (which differs by $4.2 \%$ from 1.39). As we see, the CF's associated with $q=1$ and $q=2$ are satisfactorily coincident, and therefore we conjecture that equation (13) or the one corresponding to figure 2 can be used for all values of $q(1 \leqslant q \leqslant 4)$.

4. Some other planar lattices. - The exact critical points for the isotropic Kagomé, Diced, 3-12 and Asanoha (Hemp-Leaf) lattices (see Figs. 14, 15 and 19 of reference [4]) are known [4] only for $q=2$. Thus by imposing the exact critical value $s^{(2)}$ to be equal to $s^{(q)}$ we have obtained the critical points indicated in the table I which compare well with previous $q=1$ results $[14,15]$. For the Archimedean [14] lattices we used Neal's [14] estimates for $p_{\mathrm{c}}=s^{(1)}$ to predict the critical points for $q \neq 1$ shown in the table.

5. Some first and higher neighbour lattices. The present conjecture, mainly supported by the probability-like transformation of the $s$-variable under duality, is not expected to necessarily lead to satisfactory approximate CF's for non-planar lattices. For example, for the sc lattice (fcc, bcc) $t^{(2)} \simeq 0.2181$ [20] $\left(0.1017\right.$ [20], 0.1561 [20]) hence $s^{(2)} \simeq 0.2846$ $(0.1398,0.2093)$, whereas

$$
t^{(1)}=s^{(1)} \simeq 0.2526 \pm 0.0013
$$

[21] $(0.119 \pm 0.001$ [22], $0.1785 \pm 0.0020$ [22]) ; therefore there is a discrepancy of about $13 \%(17 \%$, $17 \%$ ). Although the first and second as well as the first, second and third neighbour square and triangular lattices are not strictly planar (in the sense that they cannot be embedded in the plane; note however that their three-dimensional extension is a finite one), we have also considered them in this work since our conjecture is well verified $\left(s^{(1)}\right.$ equals $s^{(2)}$ within a $0.4 \%$ error) for the first and second neighbour square lattice (see the table). Therefore, we have used the Ising critical temperature [17, 23] to predict the $q \neq 2$ critical points for the three last lattices in the table.

In the table we present the critical points for several isotropic and homogeneous lattices and values of $q$. The estimated error bars simultaneously take into account the values available in the literature as well as the error (exactly known for the triangular and honeycomb lattices) introduced by the present conjecture. Cross-checking, by other procedures, of the critical points appearing in this table, as well as of the critical lines associated with the 4-8 lattice would be very welcome.

Acknowledgments. - We are indebted to G. Schwachheim, S. V. F. Levy and E. M. F. Curado for useful remarks.

\section{References}

[1] Kasteleyn, P. W. and Fortuin, C. M., J. Phys. Soc. Japan (suppl.) 26 (1969) 11.

[2] Śtephen, M. J., Phys. Lett. 56A (1976) 149.

[3] Burkhardt, T. W. and Southern, B. W., J. Phys. A 11 (1978) L-247;

Baxter, R. J., Temperley, H. N. V. and Ashley, S. E., Proc. R. Soc. $A 358$ (1978) 535 and references therein.
[4] Syozi, I., Phase Transitions and Critical Phenomena, vol. 1, (edited by C. Domb and M. S. Green) 1972, p. 270 and references therein.

[5] By " simple " we mean that all the coupling constants are of the ferromagnetic type.

[6] Tsallis, C. and Levy, S. V. F., J. Phys. C 13 (1980) 465; Curado, E. M. F., Tsallis, C., Levy, S. V. F. and De OliVEIRA, M. J., Phys. Rev. B 23 (1981). 
[7] Levy, S. V. F., Tsallis, C. and Curado, E. M. F., Phys. Rev. B 21 (1980) 2991 ;

Tsallis, C., J. Phys. C 14 (1981) L-85.

[8] Tsallis, C. and Levy, S. V. F., (1980), to be published.

[9] «Dual» stands for the fact that this transformation unifies the series and parallel algorithms (2) and (3); see also reference [4].

[10] Sykes, M. F. and Essam, J. W., Phys. Rev. Lett. 10 (1963) 3.

[11] Though we cannot prove it, we believe that $t^{(q)} \sim 1-L \sqrt{q}$ holds for all planar lattices, i.e. they all become, for $q \rightarrow 0$, self-dual in the sense that $s^{(q)} \rightarrow 1 / 2$.

[12] de Magalhães, A. C. N., Tsallis, C. and Schwachheim, G., J. Phys. C 14 (1981).

[13] Dean, P., Proc. Cambridge Philos. Soc. 59 (1963) 397.

[14] Neal, D. G., Proc. Cambridge Philos. Soc. 71 (1972) 97 ; the present Archimedean lattices are described by TóTH, L. F., Regular Figures (Pergamon Press) 1964.

[15] Ottavi, H., J. Physique 40 (1979) 233.

[16] Domb, C. and Potts, R. B., Proc. Roy. Soc. A210 (1951) 125

[17] Dalton, N. W. and Wood, D. W., J. Math. Phys. 10 (1969) 1271 ;

Nauenberg, M. and Nienhuis, B., Phys. Rev. Lett. 33 (1974) 944.

[18] Fan, C. and Wu, F. Y., Phys. Rev. 179 (1969) 560.

[19] Gibberd, R. W., J. Math. Phys. 10 (1969) 1026.

[20] Zinn-Justin, J., J. Physique 40 (1979) 969.

[21] De Magalhães, A. C. N., Tsallis, C. and Schwachheim, G., J. Phys. C 13 (1980) 321 and references therein.

[22] Sykes, M. F., Gaunt, D. S. and Glen, M., J. Phys. A : Math. Gen. 9 (1976) 1705.

[23] Domb, C. and Dalton, N. W., Proc. Phys. Soc. 89 (1966) 859. 